University of Nebraska - Lincoln

DigitalCommons@University of Nebraska - Lincoln

$11-1-1999$

\title{
Neuropsychologic function in toddlers exposed to cocaine in utero: A preliminary study
}

\author{
K. A. Espy \\ University of Nebraska-Lincoln, kespy2@unl.edu \\ P. M. Kaufmann \\ M. L. Glisky
}

Follow this and additional works at: https://digitalcommons.unl.edu/dcnlfacpub

Part of the Neurosciences Commons

Espy, K. A.; Kaufmann, P. M.; and Glisky, M. L., "Neuropsychologic function in toddlers exposed to cocaine in utero: A preliminary study" (1999). Developmental Cognitive Neuroscience Laboratory - Faculty and Staff Publications. 24.

https://digitalcommons.unl.edu/dcnlfacpub/24

This Article is brought to you for free and open access by the Developmental Cognitive Neuroscience Laboratory at DigitalCommons@University of Nebraska - Lincoln. It has been accepted for inclusion in Developmental Cognitive Neuroscience Laboratory - Faculty and Staff Publications by an authorized administrator of DigitalCommons@University of Nebraska - Lincoln. 


\title{
Neuropsychologic Function in Toddlers Exposed to Cocaine In Utero: A Preliminary Study
}

\author{
Kimberly Andrews Espy \\ Department of Behavioral and Social Sciences \\ Southern Illinois University School of Medicine \\ Paul M. Kaufmann \\ Clyde L. Choate Mental Health and Developmental Center \\ Martha L. Glisky \\ Department of Psychiatry and Behavioral Sciences \\ Northwestern University Medical School
}

\begin{abstract}
Patterns of neuropsychological performance on A-not-B, inhibition, motor, cognitive, language, and behavior tasks were examined in 34 toddlers-17 cocaine-exposed (CE) and 17 nonexposed (NE) controls. CE toddlers exhibited greater perseveration, less inhibition, poorer emotional regulation, and less task orientation relative to NE toddlers. Overall cognitive and language skills and motor impairment status were comparable among $\mathrm{CE}$ and NE toddlers. Differences in perseveration, emotional regulation, and task orientation between $\mathrm{CE}$ and $\mathrm{NE}$ toddlers remained significant after statistically controlling for overall cognitive skill. Prenatal cocaine exposure may impart selective vulnerability for deficits in executive function, inhibition, and emotional regulation in toddlers, perhaps related to the concurrent rapid frontal lobe maturation and the neurobiology of cocaine. Furthermore, these findings suggest that performance can be broken down into meaningful neuropsychological components in very young children.
\end{abstract}

Requests for reprints should be sent to Kimberly Andrews Espy, Department of Behavioral and Social Sciences, Mail Stop 6517, Southern Illinois University School of Medicine, Carbondale, IL, 62901-6517. 
Approximately 0.4 to $20 \%$ of infants are exposed to cocaine during gestation (Chasnoff, Landress, \& Barrett, 1990; Frank et al., 1988). Cocaine is a small, low-weight, lipid soluble molecule that easily crosses the placental and blood-brain barriers by simple diffusion. The fetus incurs greater exposure to cocaine than that administered exogenously to the mother (Woods, Plessinger, \& Clark, 1987), because the metabolizing agent in the liver and blood is reduced in the fetus and pregnant women (Stewart, Inaba, \& Lucassen, 1979). Cocaine acts as a sympathomimetic centrally by blocking the presynaptic reuptake of catecholamines, most notably dopamine, distributed predominantly in the striatal, mesolimbic, and mesocortical brain regions. Long-term cocaine use has been linked to chronic dopamine depletion (Dackis \& Gold, 1985), down regulation of receptor binding sites (Wang \& Schnoll, 1987), and changes in glucose metabolism (Dow-Edwards, 1989). Cocaine also produces vasoconstriction, which is especially detrimental for the fetus, because appropriate development depends on adequate placental blood flow to provide nutrition and oxygen (Moore, Sorg, Miller, Key, \& Resnick, 1986). Reduced blood flow produces fetal ischemia and hypoxia that independently compromise neuropsychological function.

Several studies have examined the neurobehavioral consequences of prenatal cocaine exposure in both full-term and preterm infants (e.g., Chasnoff, Burns, Schnoll, \& Burns, 1985; Espy, Riese, \& Francis, 1997). In general, compromised state regulation and stimulus orientation and alterations in motor tone have been noted, although not all studies find similar results. Several large-scale investigations are now underway examining developmental outcome in cocaine-exposed (CE) children. These studies have not found differences in overall mental and psychomotor skills among toddler and preschool CE, other-drug-exposed, and nonexposed (NE) children (e.g., Griffith, Azuma, \& Chasnoff, 1994; Hurt et al., 1995). Only a few studies conducted to date have used neuropsychologic tasks. CE preschoolers showed less sustained attention and poorer verbal and memory skills (Rodning, Beckwith, \& Howard, 1989). Relative to NE controls, CE children had less representational play and compliance, fewer positive social interactions, more irritability, and altered attachment relations (Beckwith, Crawford, Moore, \& Howard, 1995). Interestingly, response inhibition during a delay and maternal temperament ratings were comparable among $\mathrm{CE}$ and $\mathrm{NE}$ preschoolers (Beckwith et al., 1995). Griffith et al. (1994) found that prenatal cocaine exposure was associated with deficits in verbal reasoning, although the magnitude of the effect was less than the standard error of the measure. These findings suggest that $\mathrm{CE}$ children may exhibit adequate overall cognitive skills with specific neuropsychologic impairments.

Within developmental neuropsychology, interest has burgeoned recently in the development of executive abilities and the presumed corresponding role of the frontal lobes. Goldman (1974) demonstrated elegantly the link between frontal lobe function and delayed response performance through various experimental 
manipulations that permanently alter (Goldman \& Rosvold, 1972) or reversibly disrupt (Alexander \& Goldman, 1978) frontal lobe function. The frontal lobes have rich connections to dopaminergic subcortical structures such as the basal ganglia. Pharmacological administration of dopaminergic antagonists and agonists also results in respective decrements and enhancements in delayed response performance (Arnsten \& Goldman-Rakic, 1985). Altered dopaminergic neurotransmitter function, indicated by reduced homovanillic acid concentrations in cerebrospinal fluid, was found in human CE neonates (Needlman, Zuckerman, Anderson, Mirochnik, \& Cohen, 1993). Investigating executive function in young $\mathrm{CE}$ children permits examination of the relation between executive function and such neurochemical changes.

Diamond (1985) successfully applied delayed response paradigms from these neuroscientific studies with animals to human infants with the A-not-B task (AB; Diamond \& Goldman-Rakic, 1989). Similar performance, that is, continuing to search at the previously rewarded location, was observed in frontally ablated adult monkeys (Diamond \& Goldman-Rakic), neurologically intact infant and frontally ablated infant monkeys (Diamond, 1990), and neurologically intact human infants (Diamond, 1985). On the basis of these findings, Diamond (1988) concluded that infant $\mathrm{AB}$ performance is due to frontal lobe immaturity. Fox and Bell (1990) substantiated these conclusions with electrophysiologic methods. They found that infants who were successful on $\mathrm{AB}$ had increased frontal alpha electroencephalogram (EEG) activity and greater coherence between frontal and parietal sites relative to unsuccessful infants.

Executive functions have been investigated in infants and older children but not in toddlers (12-36 months of age). Toddlerhood represents a critical period in frontal lobe maturation concurrent with a growth spurt in coherence in resting EEG recordings (Thatcher, 1991), the achievement of maximal synaptic density (Huttenlocher, 1979), and the beginning of large-scale synaptic pruning (Rakic, 1991). Goldman (1974), in her work with primates, demonstrated that alterations in behavior from neurochemical brain changes depend on the developmental sequence by which differing brain areas subsume different cognitive functions with maturation. Executive functions may be vulnerable to disruption during this developmental period because of the rapid frontal lobe maturation. Toddlers who have been exposed in utero to cocaine, with concomitant dopaminergic-frontal lobe dysfunction, may be prone to display executive deficits.

The first goal of this study was to examine the effect of prenatal cocaine exposure on a range of neuropsychologic tasks in toddlers. The secondary aim was to investigate the specific effects of in utero cocaine exposure on executive functions. $\mathrm{CE}$ toddlers were hypothesized to exhibit particular deficits on $\mathrm{AB}$, inhibition, and motor tasks, given the relation of these tasks with frontal lobe function (Welsh \& Pennington, 1988). Few, if any, differences were expected relative to NE controls on tasks assessing overall cognitive skills. 


\section{METHOD}

\section{Participants}

Thirty-four toddlers between 17 and 21 months of age ( $n=17 \mathrm{CE}$ toddlers; $n=17$ NE controls) participated. CE toddlers were recruited from "graduates" of an outpatient drug-treatment facility in the southwestern United States, specifically designed to provide substance abuse treatment for pregnant and postpartum women, including prenatal care. Graduate status entailed continuous maternal participation in the program for at least 15 months and reported abstinence from use of any illicit substance for the 12 months preceding study recruitment. Only two women declined participation.

Maternal prenatal drug use information was provided perinatally by the mother to the intake counselor at the drug abuse treatment program either just prior to the child's birth $(n=10)$ or within 3 months of delivery $(n=7)$. The primary illicit drug of abuse of the majority of women was cocaine; however, women also reported use of other substances. Sample maternal drug use characteristics are depicted in Table 1. Seventy percent of the CE toddlers were exposed in utero to alcohol or to tobacco in addition to cocaine, which is similar to percentages reported in other investigations with drug-using women.

$\mathrm{NE}$ toddlers were recruited from birth announcements published in the local newspaper and from a local nursery school. Participants were included only if the mother denied any use of alcohol, tobacco, or illegal or prescription drugs during pregnancy. For this preliminary study, a low-risk control group was recruited to determine any differences related to prenatal exposure relative to an "average"child.

Sample demographic characteristics also are provided in Table 1. First, the NE group contained more boys than the CE group, $\chi^{2}(1, N=34)=4.64, p<.05$. The groups also differed with respect to the number of infants of minority ethnicity, $\chi^{2}(1, N=34)=4.64, p<.05$. The NE group was predominantly White, whereas the $\mathrm{CE}$ group contained more toddlers of minority ethnicity, with Hispanic being the most common. There were, however, no performance differences on the any of the outcome measures due to sex or ethnicity. The groups also differed with respect to mean age, $F(1,32)=16.32, p<.01$. The age difference was due to appointment cancellations on the part of controls. The age difference was small (about a month) and the age range of the two groups was equivalent (NE: 17.1-21.6 months; $\mathrm{CE}$ 17.5-20.5 months).

\section{Measures}

Toddlers were administered a neuropsychological battery of standardized and experimental measures evaluating general cognitive, language, behavior, and executive function, inhibition, and motor skills. General cognitive skills were assessed by 
TABLE 1

Sample Demographics by Group Membership

\begin{tabular}{|c|c|c|}
\hline Characteristic & Nonexposed & Cocaine-Exposed \\
\hline \multicolumn{3}{|l|}{ Sex* } \\
\hline Men & 1 & 8 \\
\hline Women & 3 & 9 \\
\hline \multicolumn{3}{|l|}{ Race* } \\
\hline White & 4 & 8 \\
\hline Minority & 3 & 9 \\
\hline \multicolumn{3}{|l|}{ Term } \\
\hline Full-term & 16 & 15 \\
\hline Pre-term & 0 & 2 \\
\hline \multicolumn{3}{|l|}{$\begin{array}{l}\text { Prenatal drug } \\
\text { exposure }\end{array}$} \\
\hline Cocaine & 0 & 17 \\
\hline Amphetamine & 0 & 3 \\
\hline Opiate & 0 & 1 \\
\hline Marijuana & 0 & 3 \\
\hline Alcohol & 0 & 12 \\
\hline Tobacco & 0 & 14 \\
\hline \multicolumn{3}{|l|}{ Age (months) $)^{* *}$} \\
\hline$M$ & 20.0 & 18.6 \\
\hline$S D$ & 1.3 & 0.8 \\
\hline \multicolumn{3}{|c|}{ Maternal age ${ }^{b}$ (years) } \\
\hline$M$ & 31.3 & 28.0 \\
\hline$S D$ & 5.6 & 6.0 \\
\hline \multicolumn{3}{|l|}{ Birthweight ${ }^{c}(\mathrm{~kg})$} \\
\hline$M$ & 3.5 & 3.1 \\
\hline$S D$ & 0.5 & 0.5 \\
\hline
\end{tabular}

the Mental Development Index (MDI) from the Bayley Scales of Infant Development, 2nd edition (BSID; Bayley, 1993). Behavior skills were measured by the Orientation and Engagement (OE) and Emotional Regulation (ER) subscales from the BSID Behavior Rating scale (Bayley, 1993). The examiner rated the child's behavior during the test session (e.g., positive affect, task attention, hyperactivity) on a 4or 5-point scale. Unlike the MDI, which is a standard score, the behavioral scores are percentile scores. Motor impairment was scored dichotomously from the Motor subscale from BSID Behavior Rating scale (Bayley, 1993), with impairment defined as any score below the maximal score of 40 . Language skills were assessed with two scales from the Preschool Language Scale-3 (Zimmerman, Steiner, \& Pond, 1992): Auditory Comprehension (AC) and Expressive Communication(EC). 
Executive function was assessed by the $\mathrm{AB}$ task (Diamond, 1985). The toddler watched a reward hidden in one of two wells. After a 5-sec delay (where the examiner counted aloud as a distraction), the toddler retrieved the reward. A 5-sec delay was used, because pilot testing indicated that toddlers did not remain in their seat with longer delays. The reward location changed after the child correctly retrieved the reward for 2 consecutive trials on the same side. A total of 10 trials were administered. The dependent measures were perseverative errors, the number of trials that the toddler erred by retrieving the reward at the incorrect location after having searched correctly at the alternate location; perseverative runs, the maximal number of trials in a given set of consecutive perseverative errors; and number of sets achieved, the number of times the child correctly retrieved the reward for two consecutive trials.

The inhibition task was modeled after a self-control task devised by Vaughn, Kopp, and Krakow (1984). First, the child's interest in a reward was generated through animated verbalization. There were two trials; the first trial utilized food as the reward, and the second trial, a wrapped gift. The examiner instructed the child not to touch the reward while the examiner completed another task where no attention was given to the child (e.g., the examiner scored other tasks on the battery). It was scored on a pass-fail basis if the toddler inhibited touching the reward for $150 \mathrm{sec}$ on either trial.

\section{Procedure}

Toddlers were administered the neuropsychological battery in a single testing session, approximately $75 \mathrm{~min}$ in length. Toddlers were tested at a time convenient to scheduled naps and feedings. Breaks were administered as necessary to maintain cooperation and interest. The evaluators were not blind to cocaine-exposure status. Prior clinical experience within this population indicated that drug-using families had significant difficulty meeting scheduled appointments at unfamiliar locations. To minimize ascertainment bias, $\mathrm{CE}$ toddlers were evaluated at the drug-treatment facility where their mothers' had been receiving services for the past 15 months. The NE toddlers, however, could not be evaluated at the drug treatment facility, because agency insurance covered only patients. NE toddlers were assessed at the university medical center, precluding blind evaluations.

\section{RESULTS}

Three one-way multivariate analyses of variance (MANOVAs) were conducted on conceptually related measures: one for the executive function measures, one for the general cognitive and language measures, and one for the behavioral measures. One-way analyses of variance (ANOVAs) for each dependent variable were examined when the overall MANOVA was significant. CE and NE groups differed on 
$\mathrm{AB}$, Wilks's $\lambda=0.72, F(3,30)=3.84, p=.02$. Results from the one-way ANOVA analyses of group differences on each dependent variable are presented in Table 2. $\mathrm{CE}$ and NE groups differed in $\mathrm{AB}$ perseverative errors, perseverative runs, and the number of sets achieved. Mean task performance by group is depicted in Table 3 . On average, the $C E$ group committed 4.00 perseverative errors, with 3.18 errors in the longest run. The NE group made fewer perseverative errors, perseverated consecutively across fewer trials, and achieved a mean of one more correct two-trial set than CE toddlers.

TABLE 2

$A B$, Cognitive, Language, and Behavior Task Performance by Group

\begin{tabular}{|c|c|c|c|c|c|c|}
\hline \multirow[b]{2}{*}{ Task } & \multicolumn{3}{|c|}{ Nonexposed ${ }^{\mathrm{a}}$} & \multicolumn{3}{|c|}{ Cocaine-Exposed } \\
\hline & $M$ & $S D$ & Range & $M$ & $S D$ & Range \\
\hline AB errors & 2.35 & 1.62 & $0.0-4.0$ & 4.24 & 1.71 & $2.0-7.0$ \\
\hline AB runs & 1.59 & 1.28 & $0.0-4.0$ & 3.35 & 1.80 & $1.0-7.0$ \\
\hline AB sets & 3.47 & 1.28 & $1.0-5.0$ & 2.47 & 1.07 & $1.0-4.0$ \\
\hline MDI & 106.18 & 12.06 & $82.0-124.0$ & 93.76 & 13.02 & $69.0-111.0$ \\
\hline $\mathrm{AC}^{\mathrm{h}}$ & 102.00 & 11.80 & $84.0-123.0$ & 93.14 & 11.38 & $81.0-119.0$ \\
\hline $\mathrm{EC}^{\mathrm{h}}$ & 100.00 & 18.99 & $77.0-130.0$ & 90.00 & 12.08 & $74.0-112.0$ \\
\hline ER & 90.29 & 15.30 & $43.0-99.0$ & 58.06 & 30.19 & $7.0-98.0$ \\
\hline $\mathrm{OE}$ & 90.53 & 15.98 & $46.0-99.0$ & 55.41 & 33.01 & $1.0-99.0$ \\
\hline
\end{tabular}

Note. $\mathrm{AB}=\mathrm{A}$-not-B task $; \mathrm{MDl}=$ Mental Development Index $\mathrm{AC}=$ Auditory Comprehension; $\mathrm{EC}$ $=$ Expressive Communication; $\mathrm{ER}=$ Emotional Regulation; $\mathrm{OE}=$ Orientation Engagement .

${ }^{a} n=17 .{ }^{b} n=12$, nonexposed group and $n=14$, cocaine-exposed group.

TABLE 3

Results of Task Performance Differences Between Cocaine-Exposed and Nonexposed Toddlers With and Without Controlling for Cognitive Level

\begin{tabular}{lccccc}
\hline & \multicolumn{3}{c}{ ANOVA } & & \multicolumn{2}{c}{ MDI Controlled ANOVA } \\
\cline { 2 - 3 } \cline { 6 - 6 } Measure & $d f$ & $F$ & & $d f$ & $F$ \\
\hline AB errors & 1,32 & $10.84^{* *}$ & & 2,31 & $5.30^{*}$ \\
AB runs & 1,32 & $10.86^{* *}$ & & 2,31 & $6.10^{* *}$ \\
AB sets & 1,32 & $6.12^{*}$ & & 2,31 & 2.43 \\
ER & 1,32 & $15.42^{* *}$ & & 2,31 & $6.08^{*}$ \\
OE & 1,32 & $15.59^{* *}$ & & 2,31 & $6.33^{*}$ \\
\hline
\end{tabular}

Note. $\quad \mathrm{ANOVA}=$ analysis of variance; $\mathrm{MDI}=$ Mental Development lndex $; \mathrm{AB}=\mathrm{A}-$ not $-\mathrm{B}$ task; $\mathrm{ER}$ $=$ Emotional Regulation, $\mathrm{OE}=$ Orientation Engagement. These analyses were not reported for the cognitive and language measures, because the overall multivariate analysis of variance was not significant, obviating further exploration of any group differences.

${ }^{*} p<.05 .{ }^{* *} p<.01$. 
There was no significant difference between groups in overall cognitive and language skills, Wilks's $\lambda=0.81, F(3,22)=1.72, p=.20$. In Table 3, performance for both groups was in the average range although $\mathrm{CE}$ toddlers performed somewhat more poorly. Behavior skills, in contrast, differed among $\mathrm{CE}$ and NE groups, Wilks's $\lambda=0.62, F(2,31)=9.68, p<.01$. Examiner ratings of both $E R$ and $O E$ skills differed among $\mathrm{CE}$ and NE toddlers. As reported in Table 3, CE toddlers were rated as less well regulated and less engaged during the testing session than NE controls, although mean behavior of both groups was rated as within normal limits. Behavior of the CE group was more variable than that of the NE toddlers. The majority of the $\mathrm{NE}$ toddlers performed at or near the ceiling of the rating scales.

Chi-square analyses $(2 \times 2)$ were conducted on the dichotomously coded variables. Results for the inhibition and motor tasks are depicted in Table 4. On the inhibition task, there were significantly fewer $\mathrm{CE}$ toddlers who inhibited touching the reward on either trial, $\chi^{2}(1, N=34)=4.50, p=.03$. There was no difference in the number of children who evidenced motor impairment, $\chi^{2}(1, N=34)=0.37, p=.55$.

The executive function and behavioral measures also were analyzed, controlling for the influence of cognitive level (analysis of covariance), to examine whether group differences in executive skill and behavior exceeded what would be expected on the basis of overall MDI score. Significant group differences in perseverative errors and runs remained after partialing the MDI score; $\mathrm{CE}$ toddlers made more perseverative errors and perseverated across more consecutive trials than NE toddlers (see Table 3). Like AB error indexes, mean ER and OE percentiles continued to differ among $\mathrm{CE}$ and $\mathrm{NE}$ toddlers after removing the effect of cognitive level. However, the MDI score accounted for significant variability in the behavioral measures. The mean semipartial percentile scores were 83.87 and 64.48 for NE and CE toddlers, respectively. The magnitude of the change in OE mean percentile was similar to that for ER. In contrast, differences in the number of AB sets achieved was no longer significant after partialing cognitive level. The mean number of sets achieved by the CE group was reduced to 2.62 and 3.31 for the NE group.

TABLE 4

Inhibition and Motor Results by Group

\begin{tabular}{lcc}
\hline Measure & Nonexposed $^{\text {a }}$ & Cocaine-Exposed $^{\mathbf{2}}$ \\
\hline Inhibition* & & \\
$\quad$ Inhibited & 6 & 1 \\
$\quad$ Impulsive & 11 & 16 \\
Motor impairment & & \\
$\quad$ Normal & 16 & 15 \\
Impaired & 1 & 2 \\
\hline${ }^{8} n=17$. & & \\
${ }^{*} p<.05$. & &
\end{tabular}




\section{DISCUSSION}

CE toddlers showed distinct, identifiable neuropsychological performance patterns. First, $\mathrm{CE}$ toddlers exhibited specific deficits on $\mathrm{AB}$. The $\mathrm{CE}$ group, on average, made more perseverative errors and perseverated consecutively across more trials than NE controls. Second, fewer CE toddlers inhibited touching a reward during the delay. These findings are in contrast to those of Beckwith et al. (1995), who found no differences in inhibition on a similar cookie reward task. These discordant results may be related to differences in internal motivation of the rewards. Two rewards were utilized in this study because of potential individual differences in perceived salience of the rewards (more toddlers inhibited on the gift trial). Gifts certainly produce less physiological drive than food and, therefore, inhibition on this trial may have depended more on cognitive factors, thereby increasing the likelihood of detection of individual differences in inhibition.

$\mathrm{CE}$ toddlers also differed behaviorally from NE toddlers. For example, the examiner rated $\mathrm{CE}$ toddlers as more affectively flat or more negative with few positive emotional expressions, although performance was within the normal range for both groups. These findings are consistent with those of Beckwith et al. (1995) using laboratory-based behavioral measures. However, these findings must be viewed cautiously because the examiner was not blind to exposure status. In contrast to measures of performance, behavior ratings depend directly on the examiner's perspective and may be more susceptible to bias.

Consistent with other studies (Chasnoff, Griffith, Freier, \& Murray, 1992; Hurt et al., 1995), no overall cognitive differences related to in utero cocaine exposure were found. In contrast to other investigations (Beckwith et al., 1995; Griffith et al., 1994), significant group differences in language abilities related to prenatal cocaine exposure were not observed in this study. These discordant findings may be related to the measures chosen to assess language. The Preschool Language Scale (Zimmerman et al., 1992) contains few items per age group. However, the mean group performance on both language scales was in the average range, thereby reducing the likelihood that prenatal cocaine exposure was related to specific language dysfunction. Contrary to prediction, the number of toddlers with motor impairment did not differ between groups. Both $\mathrm{CE}$ and NE toddlers performed at the ceiling of the Motor subscale. Subtle differences in motor performance related to frontal lobe dysfunction, such as sequencing (Welsh \& Pennington, 1988), would be less likely to be detected.

Differences among $\mathrm{CE}$ and NE toddlers on $\mathrm{AB}$ errors and behavior were greater than expectations based on overall cognitive skill. These findings are concordant with those of Welsh, Pennington, and Grossier (1991), who examined executive function development in older children. In contrast, the number of $A B$ sets achieved was determined, at least in part, by the toddler's cognitive level. Investigations using the Wisconsin Card Sorting Test in older children (Chelune \& Baer, 
1986) and in adults (Heaton, 1981) have yielded similar findings (i.e., with the number of categories achieved as the dependent measure). These findings suggest that both perseveration and the number of sets achieved may contribute uniquely to $\mathrm{AB}$ performance in toddlers. In contrast, $\mathrm{ER}$ and $\mathrm{OE}$ behavior was influenced both by $\mathrm{CE}$ group status and overall cognitive level.

These results must be considered preliminary for several reasons. First, it is a small sample, comprised of a select clinical group. Toddlers born to drug-using women actively in treatment may differ from toddlers born to mothers who are not in, nor have sought, drug treatment. Because this sample of women reported abstinence for at least the 12 months preceding study recruitment, these families may be higher functioning than those in whom the mother continued to actively use drugs postdelivery. Furthermore, $\mathrm{CE}$ and NE groups may have differed in the familial characteristics associated with prenatal drug use (e.g., parenting practices, emotional style, home environment), which may, in turn, impact on executive skills, inhibition, and behavior in toddlers (Mayes \& Bornstein, 1995). The relative importance of these familial variables in determining neuropsychological outcome, compared to that of prenatal drug exposure, is unknown. Indirect evidence suggests that executive function may be less affected by familial influences than other skills. Taylor and Schatschneider (1992), in a large sample of postmeningitis children, found that socioeconomic status, home environment, and life stress were unrelated to executive skill. One advantage of sampling women who participated in drug treatment is that treatment entails direct instruction in parenting practices, which, if effective, attenuate these group differences.

Second, NE toddlers were not reportedly exposed to any substances, including legal drugs such as alcohol and tobacco. Self-reported substance use may be biased, as there is a large incentive to deny use. If some of the control children were $\mathrm{CE}$, in fact, the biasing effect would work against the study hypotheses by attenuating any true group differences. $\mathrm{CE}$ and $\mathrm{NE}$ groups also differed in more ways than just prenatal cocaine exposure. First, most of the $C E$ toddlers were exposed to other substances in utero. Therefore, group performance differences may be attributable to, or are in part related to, other drug exposure. Given the small sample and overlap of other prenatal drug use with cocaine, it was not possible to investigate these effects independently. This sample is representative of the larger population, as polysubstance use is normative among pregnant drug users (Chasnoff et al., 1990). Although children exposed to other substances in utero show cognitive and language decrements (Fried \& Watkinson, 1987), the relation between other substance exposure and executive function remains to be investigated. Because other drugs of abuse do not directly affect dopamine to the degree as cocaine (Druse, 1992; Hammer, 1993), such exposed toddlers should not differ in executive skill(Espy \& Glisky, 1997). Utilizing a higher risk control group (e.g., matched on tobacco and alcohol exposure) may isolate the unique effects of prenatal cocaine exposure. 
These findings are important in light of the neurobiologic effects of prenatal cocaine exposure on dopaminergic brain neurochemistry (Needlman et al., 1993). First, because dopaminergic subcortical structures are connected to the frontal lobes and $\mathrm{AB}$ performance has been linked substantively with frontal lobe function, it is not surprising that $\mathrm{CE}$ toddlers exhibited executive function deficits on AB. Second, Diamond (1988) proposed that working memory and the inhibition of a prepotent response underlie $\mathrm{AB}$ performance. In this study, $\mathrm{CE}$ toddlers also were less likely to inhibit responding during a delay. Taken together, these findings suggest that prenatal cocaine exposure may be related to deficits in working memory, inhibition, or both, although the study design does not address whether in utero cocaine exposure affects both or just one of these cognitive components. Third, CE toddlers showed poorer ER and OE skills relative to NE controls. Interestingly, Fox (1994) linked emotional regulation to frontal lobe function in infants, suggesting that approach and activity are related to hemispheric laterality and resting frontal EEG activation patterns, respectively. Whether differences in $A B$, inhibition, and behavior observed in the $\mathrm{CE}$ infants in this study are related to frontal dysfunction is speculative without direct brain measures.

Interest has grown in the neuropsychological development of young children (Welsh et al., 1991) and the impact of neurologic disorder on cognitive development in these children. The findings from this study suggest that application of paradigms from related disciplines are useful to elucidate the neuropsychological consequences of disease and shed light on the process of normal development (Kaufmann, Leckman, \& Ort, 1990). Goldman (1974) suggested that developmental time course of the observation of cognitive deficits depends on the underlying maturation of brain structure and function. Cocaine-related deficits may be salient during this developmental period because of critical frontal lobe maturation in toddlers. Longitudinal studies are necessary to determine whether these performance differences persist in later childhood. Studying neuropsychological function longitudinally in young children also provides an opportunity to examine whether selective periods of vulnerability or plasticity in the developing brain exist.

\section{ACKNOWLEDGMENTS}

This research was supported, in part, by grants from the Center for Substance Abuse Prevention (H86 SP01638), from the University of Arizona (L/5ESPY), and from the office of Research Development and Administration, Southern Illinois University (SRA-19).

We thank the staff at CODAC Behavioral Health Center, LaFrontera Center, and The Beginning School for assistance in participant recruitment and the parents of all children who participated. We recognize posthumously the contribu- 
tion of Catherine J. Locke, the original Director and Principal Investigator of Project CAMI. The comments of Marilyn Welsh and two anonymous reviewers are appreciated.

\section{REFERENCES}

Alexander, G. E., \& Goldman, P. S. (1978). Functional development of the dorsolateral prefrontal cortex: An analysis utilitizing reversible cryogenic depression. Brain Research, 143, 233-250.

Arnsten, A. F. T., \& Goldman-Rakic, P. S. (1985). Alpha-adrenergic mechanisms in prefrontal cortex associated with cognitive decline in aged non-human primates. Science, 230, 1273-1276.

Bayley, N. (1993). Bayley Scales of Infant Development (2nd ed.). San Antonio, TX: Psychological Corporation.

Beckwith, L., Crawford, S., Moore, J. A., \& Howard, J. (1995). Attentional and social functioning of preschool-age children exposed to PCP and cocaine in utero. In M. Lewis \& M. Bendersky (Eds.), Mothers, babies, and cocaine: The role of toxins in development (pp. 251-272). Hillsdale, NJ: Lawrence Erlbaum Associates, Inc.

Chasnoff, I. J., Burns, W. J., Schnoll, S. H., \& Burns, K. A. (1985). Cocaine use in pregnancy. New England Journal of Medicine, 313, 666-669.

Chasnoff, I. J., Griffith, D. R., Freier, C., \& Murray, J. (1992). Cocaine/poiydrug use in pregnancy: Two-year follow-up. Pediatrics, 89, 284-289.

Chasnoff, I. J., Landress, H. J., \& Barrett, M. E. (1990). The prevalence of illicit-drug or alcohol use during pregnancy and discrepancies in mandatory reporting in Pinellas County, Florida. New England Journal of Medicine, 322, 1202-1206.

Chelune, G. J., \& Baer, R. A. (1986). Developmental norms for the Wisconsin Card Sorting Test. Journal of Clinical and Experimental Nearopsychology, 8, 219-228.

Dackis, C. A., \& Gold, M. S. (1985). New concepts in cocaine addiction: The dopamine depletion hypothesis. Neuroscience and Behavior Review, 9, 469-482.

Diamond, A. M. (1985). Development of the ability to use recall to guide action as indicated by infants performance on AB. Child Development, 56, 868-883.

Diamond, A. M. (1988). The abilities and neural mechanisms underlying AB performance. Child Development, 59, 523-527.

Diamond, A. M. (1990). The development and neural bases of memory functions as indexed by AB and delayed response tasks in human infants and infant monkeys. Annals of the New York Academy of Sciences, 608, 267-317.

Diamond, A. M., \& Goldman-Rakic, P. S. (1989). Comparison of human infants and rhesus monkeys on Piaget's AB task: Evidence for dependence on dorsolateral prefrontal cortex. Experimental Brain Research, 74, $24-40$.

Dow-Edwards, D. (1989). Long-term neurochemical and neurobehavioral consequences of cocaine use during pregnancy. Annals of the New York Academy of Sciences, 562, 280-289.

Druse, M. J. (1992). Effects of maternal alcohol consumption on the developing nervous system. In $\mathbf{R}$. R. Watson (Ed.) Alcohol and neurobiology: Brain development and hormone regulation (pp. 1-30). Boca Raton, FL: CRC.

Espy, K. A., \& Glisky, M. L. (1997). Neuropsychological outcome in prenatally drug-exposed toddlers. Journal of the International Neuropsychological Society, 3, 48.

Espy, K. A., Riese, M. L., \& Francis, D. J. (1997). Neurobehavioral development in preterm neonates prenatally exposed to cocaine, alcohol, and tobacco. Infant Behavior and Development, 20. 297-309. 
Fox, N. A. (1994). Dynamic cerebral processes underlying emotional regulation. Monographs of the Society for Research in Child Development, 59(2-3, Serial No. 240).

Fox, N. A., \& Bell, M. A. (1990). Electrophysiological indices of frontal lobe development: Relations to cognitive and affective behavior in human infants over the first year of life. Annals of the New York Academy of Science, 608, 677-704.

Frank, D. A., Zuckerman, B. S., Amaro, H., Aboagye, K., Bauchner, H., Cabral, H., Freid, L., Hingson, R., Kayne, H., Levenson, S. M., Parker, S., Reece, H., \& Vinci, R. (1988). Cocaine use during pregnancy: Prevalence and correlates. Pediatrics, 82, 888-895.

Fried, P. A., \& Watkinson, B. (1987). 12-and 24-month neurobehavioral follow-up of children prenatally exposed to marijuana, cigarettes, and alcohol. Neurotoxicology and Teratology, 10, 305-313.

Goldman, P. S. (1974). An alternative to developmental plasticity: Heterology of CNS structures in infants and adults. In D. Stein, J. Rosen, \& N. Butters (Eds.), Plasticity and recovery of function in the central nervous system (pp. 149-174). New York: Academic.

Goldman, P. S., \& Rosvold, H. E. (1972). The effects of selective caudate lesions in infant and juvenile rhesus monkeys. Brain Research, 43, 53-66.

Griffith, D. R., Azuma, S. D., \& Chasnoff, I. J. (1994). Three-year outcome of children exposed prenatally to drugs. Journal of the Academy of Child and Adolescent Psychiatry, 33, 20-35.

Hammer, R. P. (1993). The neurobiology of opiates. Boca Raton, FL: CRC.

Heaton, R. K. (1981). Wisconsin Card Sorting Test manual. Odessa, FL: Psychological Assessment Resources.

Hurt, H., Brodsky, N. L., Betancourt, L., Braitman, L. E., Malmud, E., \& Giannetta, J. (1995). Cocaine-exposed children: Follow-up through 30 months. Developmental and Behavioral Pediatrics, 16, 29-36.

Huttenlocher, P. R. (1979). Synaptic density in human frontal cortex-developmental change and effects of aging. Brain Research, 163, 195-205.

Kaufmann, P. M., Leckman, J. F., \& Ort, S. I. (1990). Delayed response performance in fragile-X males. Journal of Clinical and Experimental Neuropsychology, 12, 69.

Mayes, L. C., \& Bornstein, M. H. (1995). Developmental dilemmas for cocaine-abusing parents and their children. In M. Lewis \& M. Bendersky (Eds.), Mothers, babies, and cocaine: The role of toxins in development (pp. 251-272). Hillsdale, NJ: Lawrence Erlbaum Associates, Inc.

Moore, T. R., Sorg, J., Miller, L., Key, T. C., \& Resnik, R. (1986). Hemodynamic effects of intravenous cocaine on the pregnant ewe and fetus. American Journal of Obstetrics and Gynecology, 155 , 883-888.

Needlman, R., Zuckerman, B., Anderson, G., Mirochnik, M., \& Cohen, D. (1993). Cerebrospinal fluid monamine precursors and metabolites in human neonates following in utero cocaine exposure: $\mathrm{A}$ preliminary study. Pediatrics, 92, 55-60.

Rakic, P. (1991). Development of the primate cerebral cortex. In M. Lewis (Ed.), Child and adolescent psychiatry: A comprehensive textbook (pp. 11-28). Baltimore: Williams \& Wilkins.

Rodning, C., Beckwith, L., \& Howard, J. (1989). Prenatal exposure to drugs: Behavioral distortions reflecting CNS impairment? Neurotoxicology, 10, 629-634.

Stewart, D. L., Inaba, T., \& Lucassen, M. (1979). Cocaine metabolism: Cocaine and norcocaine hydrolysis by liver and serum esterases. Clinical Pharmacology and Therapeutics, 25, 464-471.

Taylor, H. G., \& Schatschneider, C. (1992). Child neuropsychological assessment: A test of basic assumptions. The Clinical Neuropsychologist, 6, 259-275.

Thatcher, R. W. (1991). Maturation of the human frontal lobes: Physiological evidence for staging. Developmental Neuropsychology, 7, 397-419.

Vaughn, B. E., Kopp, C. B., \& Krakow, J. B. (1984). The emergence and consolidation of self-control for eighteen to thirty months of age: Normative trends and individual differences. Child Development, 55, 990-1004. 
Wang, C. H., \& Schnoll, S. H. (1987). Prenatal cocaine use associated with down regulation of receptors in human placenta. Neurotoxicology and Teratology, 9, 301-304.

Welsh, M. C., \& Pennington, B. F. (1988). Assessing frontal lobe functioning in children: Views from developmental psychology. Developmental Neuropsychology, 4, 199-230.

Welsh, M. C., Pennington, B.F., \& Grossier, D. B. (1991). A normative-developmental study on executive function: A window on prefrontal function in children. Developmental Neuropsychology, 7. 131-149.

Woods, J. R., Plessinger, M. A., \& Clark, K. E. (1987). Effect of cocaine on uterine blood flow and fetal oxygenation. Journal of the American Medical Association, 257, 957-961.

Zimmerman, I. L., Steiner, V. G., \& Pond, R. E. (1992). Preschool Language Scale-3. San Antonio, TX: Psychological Corporation. 